\title{
Bird diversity and ethno-ornithological knowledge of local people in Ciletuh-Palabuhanratu Geopark, Sukabumi, West Java, Indonesia
}

\author{
JOHAN ISKANDAR ${ }^{1,2,3, \bullet}$, SYAHRAS FATHIN A ${ }^{3}$, HASNA SILMI $\mathbf{R}^{1}$, TEGUH HUSODO ${ }^{1,2,3}$, \\ INDRI WULANDARI ${ }^{1,2,3}$, ERRI N. MEGANTARA ${ }^{1,2,3}$, RUHYAT PARTASASMITA ${ }^{1,2,3}$, SYA SYA SHANIDA ${ }^{3}$ \\ ${ }^{1}$ Department of Biology, Faculty of Mathematics and Natural Sciences, Universitas Padjadjaran. Jl. Raya Bandung-Sumedang Km. 21, Jatinangor, \\ Sumedang 45363, West Java, Indonesia. Tel.: +62-22-7796412 ext. 104, Fax.: +62-22-7794545. "email: johan.iskandar@unpad.ac.id \\ ${ }^{2}$ Program in Environmental Science, School of Graduates, Universitas Padjadjaran. Jl. Sekeloa, Coblong, Bandung 40134, West Java, Indonesia \\ ${ }^{3}$ Center of Environment and Sustainable Science (CESS), Directorate of Research, Community Services and Innovation, Universitas Padjadjaran. \\ J1. Raya Bandung-Sumedang Km. 21, Jatinangor, Sumedang 45363, West Java, Indonesia
}

Manuscript received: 12 July 2021. Revision accepted: 24 July 2021.

\begin{abstract}
Iskandar J, Fathin AS, Silmi RH, Husodo T, Wulandari I, Megantara EN, Partasasmita R, Shanida SS. 2021. Bird diversity and ethno-ornithological knowledge of local people in Ciletuh-Palabuhanratu Geopark, Sukabumi, West Java, Indonesia. Biodiversitas 22: 3409-3422. Birds have ecological and social functions, and are common to be used as bioindicators of environmental changes. Birds are very important consideration when establishing conservation areas, including geopark which has uniqueness in terms of geological, biological and cultural diversities. This study aimed to investigate the bird diversity in Ciletuh-Palabuhanratu Geopark, West Java, Indonesia and as well as to reveal the local knowledge on bird species and folk classification, functions and utilization of birds among the village people. Mixed methods of quantitative and qualitative approaches were applied in this study, while some techniques, including semi-structured interviews, structured interviews, and bird observation on transect and spotlighting were employed to collect the primary data. In total, there were 121 bird species of 47 families of which 88 species were recorded during the field observation and 79 species were documented through the interviews with respondents. Among them, 12 species are listed as having high conservation status under IUCN, 17 species are listed as Appendix II (CITES), and 21 species are listed by Regulation of the Minister of Environment and Forestry of the Republic Indonesia No. P 106/2018 on Protected Plant and Animal Species. The local community has profound knowledge of birds species, folk classification, ecological, socio-cultural, and socio-economic functions. In the past, the village people had traditional beliefs and myths on birds, resulting in some sacred birds had been traditionally conserved. Nowadays, these are no longer strongly existed, and consequently, many birds have been hunted by using different methods of hunting birds, both traditional and modern for various purposes, including for consumption, bird keeping, and trading to obtain cash. Therefore, bird conservation efforts that involve the community are really needed in this area as the strategy of sustainable management of Ciletuh-Palabuhanratu Geopark.
\end{abstract}

Keywords: Avifauna, Ciletuh-Palabuhanratu Geopark, local knowledge

\section{INTRODUCTION}

Birds play an important role in ecological and socioeconomic functions. In ecological views, Philpott et al. (2009) and Sekercioglu (2012) stated that birds perform essential services for regulating ecosystem health, such as seeds dispersals, pollination of tropical plants and pest control. Birds can also suppress arthropod density, so that it increases agricultural productivity, for example, palm oil yield (Maas et al. 2015). Yet, in some countries, including Indonesia, birds are mostly recognized for utilitarian uses such as food, jewelry, decorative accessories, and traditional medicine (Alves et al. 2013).

Birds are good bio-indicators for environmental changes resulting from land use change. Birds are very sensitive to changes in the ecosystem so they can be a strong indicator of the species richness and of the presence of certain plant species. The use of birds as bio-indicators can explain to what extent human activities have changed habitat quality and how this change has affected biodiversity. Moreover, birds play important roles in the ecosystem due to their contribution to seed dispersal and pollination (Bibby et al. 1992). Philpott et al. (2009) and Sekercioglu (2012) said that birds perform essential services for regulating ecosystem health, such as seed dispersals, pollination of tropical plants, and pest control. Birds have beneficial impacts on agroforestry systems because they can suppress anthropoid density, thus increasing palm oil yield (Maas et al 2015).

Some recorded bird species have become rare or locally extinct (Iskandar et al. 2016), although some others have been endured in the last several decades. Some factors have a negative impact on the birds, especially those involving loss and degradation of habitat (including the intensive use of pesticides in agriculture) and the excessive exploitation of bird population. Many birds can be traded at high prices, resulting in the high pressures from both hunting and catching bird species which have been shown to affect their natural populations (Iskandar 2016). Consequently, the bird populations have dramatically decreased with the main factor influencing bird populations is human activity (Alves et al. 2013). Due to the main source of problems of 
threatened birds, we cannot talk about bird conservation without incorporating human dimension aspects (Alves 2012; Alves et al. 2013; Alves and Souto 2015).

The Ciletuh-Palabuhanratu Geopark, Sukabumi Regency, West Java, Indonesia has been officially declared as a geopark on December 22, 2015 (Andriany et al. 2016; Yanuar et al. 2018; Wulandari et al. 2021). The ultimate goal of the designation of the geopark is sustainable management and conservation of the geological, biological, and cultural diversities in Ciletuh-Pelabuhanratu region (UNESCO 2017). The geopark covers approximately 128,000 hectares, including 74 villages across eight subdistricts (i.e., Ciemas, Cisolok, Cikakak, Palabuanratu, Simpenan, Ciracap, Surade and Waluran). The CiletuhPalabuhanratu Geopark is characterized by rare geological diversity classified into three zones: uplifted rock subduction zones, the Jampang Plateaus landscape, and ancient magmatic zone shift and forearm evolution (UNESCO 2017). In addition, it has rich biodiversity and local cultural diversity.

Previous biodiversity studies had been conducted in Ciletuh-Palabuhanratu Geopark, including vertical stratification of bird community in Cikepuh Wildlife Reserve (Dinanti et al. 2018). This study is essential considering the lack of research on birds in CiletuhPalabuhanratu, especially in Ciemas sub-district, based on ecological and local knowledge. The objectives of this study are to investigate the bird diversity, local knowledge on the bird species and folk classification, and the bird functions and utilizations in Ciletuh-Palabuhanratu Geopark, Ciemas Sub-district, Sukabumi, West Java.

\section{MATERIALS AND METHODS}

\section{Study area and period}

Ciletuh-Palabuhanratu Geopark is located in Sukabumi District, West Java Province, Indonesia, encompassing eight sub-districts, including Ciemas, Cisolok, Surade Cikakak, Palabuhanratu, Simpenan, Ciracap, and Waluran. The areas of the geopark were established in September 2016 through the Decree of the Government of West Java No. 556/Kep. 941-Rek/2016 (Wulandari et al. 2018). The area is bordered by Banten Province to the west, while to the south by the open sea of the Indian Ocean, and to the north and east, it is bordered with Bogor and Cianjur District, respectively. The area is located about the exact distance from Jakarta (the capital city of Indonesia) and Bandung (the provincial capital), 125 kilometers away (Haryanto and Sudjradjat 2018).

This study was conducted in February 2017 and February 2018 in the Ciemas Subdistrict, Sukabumi, West Java. The Ciemas Subdistrict is bordered by the Simpenan Subdistrict (north), the Indian Ocean (south and west), and the Ciracap Subdistrict (east). Ciemas Subdistrict is at an altitude of $10-400 \mathrm{~m}$ asl. Ciemas Subdistrict consists of nine villages, namely Tamanjaya, Mekarjaya, Ciemas,
Girimukti, Mekarsakti, Cibenda, Mandrajaya, Ciwaru, and Sidamulya.

We selected observation sites in three villages located in the Amphitheater area, namely Tamanjaya, Girimukti, and Mandrajaya Village. The selection of these three sites was based on the existence of remnant forest and assuming those sites representing the Ciletuh-Palabuhanratu Geopark in general. In addition, we selected the Cingaleng and Cipicung hamlets, Girimukti Village, as population samples for interviews. The selection of those hamlets was because the village is close to remnant forests, and many people were hunting birds compared to other villages. Hence, the community knew birds more than other communities.

\section{Procedures}

The data collection used a mixed-method of qualitative and quantitative using an ethno-ornithological approach (Albuquerque et al. 2014; Iskandar 2018). The researchers study the viewpoint of local communities who have traditional ecological knowledge of birds embedded with their culture (Berkes 2012; Iskandar and Iskandar 2021). Some techniques were applied in this study for data collection, including field observation, semi-structured and structured interviews (Creswell 2009).

The semi-structured interview was conducted to document bird diversity based on local knowledge, the role of birds, bird utilizations, and bird hunting. The semistructured interview was conducted through in-depth interviews with local experts purposively selected (Iskandar et al. 2016; Iskandar 2018). The guidelines for interviews were made previously and developed during the interview (Husodo et al. 2019). Informants were chosen through the snowball sampling technique by selecting one local expert with excellent knowledge, then based on this initial informant, some other local experts were recognized and purposively selected as informants (Albuquerque et al. 2014). The informants in this study were local government (village head/kades and the staff) as a base informant, elders (sesepuh), animal hunters, shamans (dukun), and animal traders (Partasasmita et al. 2016).

The structured interview was conducted to determine the knowledge regarding birds, bird naming, bird hunting, and bird-related myths (community-based conservation). The number of respondents was determined based on the Lynch et al. (1974) formula as following:

$$
\mathrm{n}=\frac{\mathrm{N} \cdot \mathrm{Z}^{2} \cdot \mathrm{P}(1-\mathrm{P})}{\mathrm{N} \cdot \mathrm{d}^{2}+\mathrm{Z}^{2}(1-\mathrm{P})}
$$

Where:

$\begin{array}{ll}\mathrm{n} & : \text { Number of samples } \\ \mathrm{N} & : \text { Population size } \\ \mathrm{Z} & \text { : Number of normal variables }(1.96) \\ \mathrm{P} & : \text { The greatest possible proportion }(0.50) \\ \mathrm{d} & : \text { Error }(0.10)\end{array}$




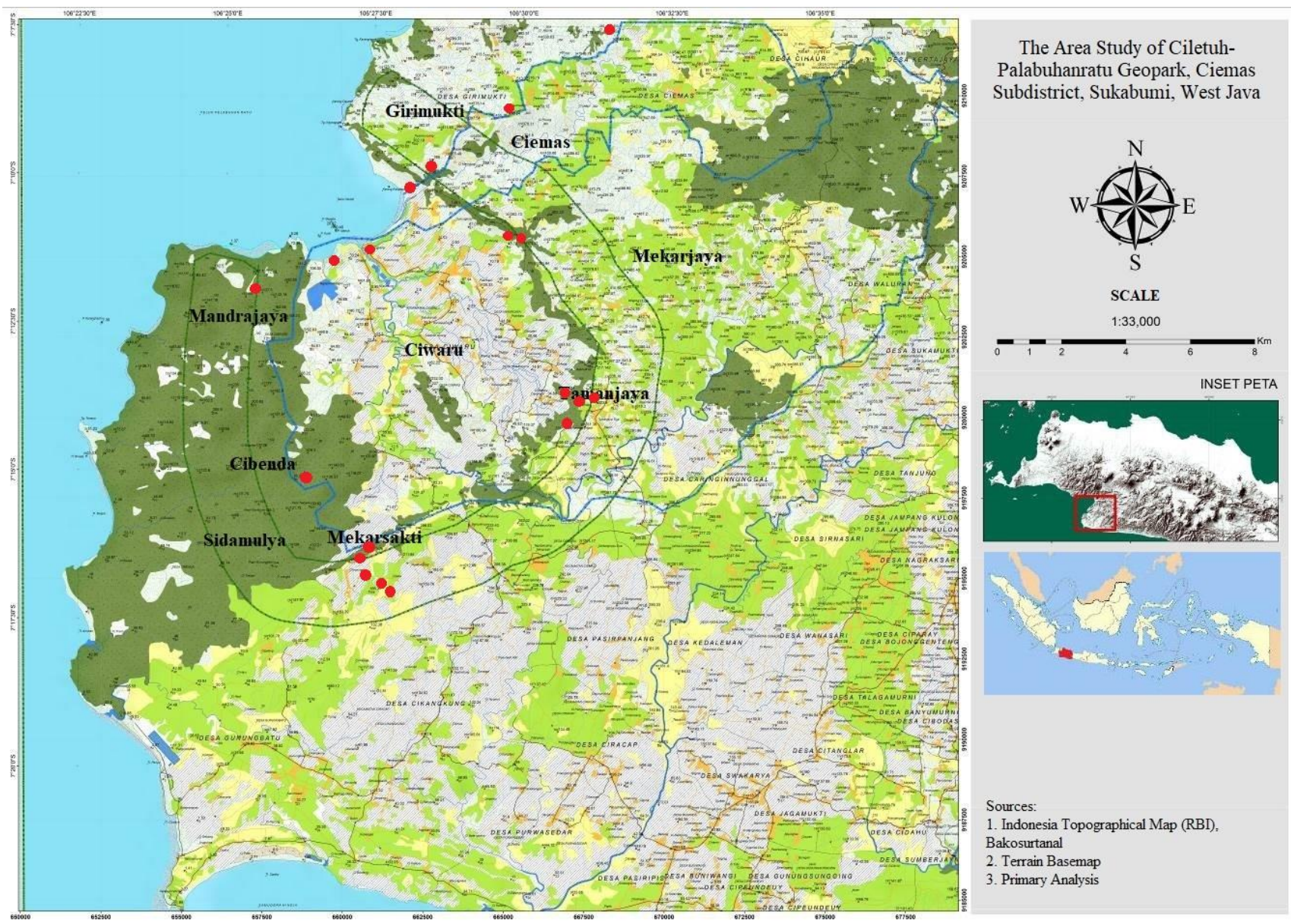

Figure 1. Sampling sites in Ciletuh-Palabuhanratu Geopark Ciomas Sub-district, Sukabumi District, West Java, Indonesia (48 M $66301100 \mathrm{~m}$ E $9201983.71 \mathrm{~m}$

The respondents were selected proportionally using random sampling which resulted in 84 household heads of 645 household heads in Cingaleng and Cipicung hamlets. Respondents selected were the household heads, assuming they represented their family knowledge (Wulandari et al. 2021). Structured interviews were undertaken with each household head or respondent using a questionnaire (Newing et al. 2011; Iskandar 2012).

Field observation was conducted using transect and spotlighting methods. The transect method in the morning between 05.30 and $11.00 \mathrm{a} . \mathrm{m}$. and in the afternoon between 02.00 and 05.00 p.m. Total day of observation is 14 days ( 140 observation hours). Data collection was conducted on foot (approximately $2 \mathrm{~km} /$ hour) by recording bird species either they were visible and only audible. This transect method was based on the proportion of the area observed and the time available for observation. The spotlighting method was carried out to record nocturnal species and conducted between 07.00 and 11.00 p.m. using a flashlight and headlamp. Night-watching was repeated on the track that has been traversed in the morning or carried out at locations considered to have the potential to encounter the birds (Husodo et al. 2020). We use the field guide by Mackinnon (2010) to identify species in the interviews and field observation.

\section{Data analysis}

The qualitative data was analyzed by cross-checking, summarizing and synthesizing as the basis for developing narrative writing (Newing et al. 2011; Partasasmita et al. 2019; Iskandar and Iskandar 2021). Cross-checking data was conducted using the triangulation method using the information obtained from informants collected in the interviews and field observation. While the quantitative data were analyzed using descriptive statistics, including the frequency and proportion of respondents' answers on particular issues, as follows (Wulandari et al. 2019; Iskandar and Iskandar 2021):

$$
\mathrm{Fi}=\frac{\mathrm{ni}}{\mathrm{N}} x 100 \%
$$

Where:

Fi : Frequency of respondent's answer to question i

$\mathrm{N}$ : Total respondent

The results of the statistical analysis are interpreted and narrated with descriptive and evaluated analyses. 


\section{RESULTS AND DISCUSSION}

\section{Bird diversity}

The bird diversity in the studied area in Ciemas Subdistrict, Ciletuh-Palabuhanratu Geopark was 121 species belong to 47 families, in which 88 species were recorded in the field observation and 79 species were documented in the interviews (Tables 1 and 2). Some species that were recorded in this study are presented in Figure 2.

Among the 121 species recorded, two species are categorized as Endangered (EN) status according to the International Union for Conservation of Nature (IUCN), four species as Near Threatened (NT), six species as Vulnerable (VU), and other species as Least Concern (LC). Two species with Endangered status included Javan Hawkeagle (Nisaetus bartelsii) and Java Sparrow (Padda oryzivora). According to the Convention on International Trade in Endangered Species (CITES, 2014), 17 species are listed as Appendix II and 21 species are listed by The Regulation of the Minister of Environment and Forestry of the Republic of Indonesia No. P 106/2018 on Protected Plant and Animal Species.

Since the Ciletuh-Palabuhanratu Geopark has a high diversity of birds, including endangered species and protected birds based on Indonesian regulations, this geopark plays an important role not only for the geological distinctive characteristic but also for bird conservation.

It can be seen from Table 1 that bird diversity recorded in Ciletuh-Palabuhanratu Geopark is higher than that recorded in other sites in West Java. Bird diversity in Ciletuh-Palabuhanratu Geopark has recorded 121 species of 47 families, whereas in Karangwangi village, Cianjur, it was recorded 59 species of 28 families (Iskandar et al. 2016), Pangalengan Bandung recorded 60 species of 29 families (Withaningsih et al. 2020), and Kertasari Bandung of upper Citarum watershed recorded 29 species of 17 families (Hakim et al. 2020).

There are some factors that may cause the CiletuhPalabuhanratu-Geoparpark to have a high diversity of bird, including the size of area, habitat diversity, and local environmental conditions. It was widely argued that there is a positive correlation between size of area and diversity of birds. This study in Ciletuh-Palabuhanratu-Geopark was undertaken in large area that covered three villages which is larger than the study area in Karangwangi, Pangalengan, and Kertasari which were undertaken in one or two villages. As a result, the diversity of birds recorded in Ciletuh-Palabuhanratu Geopark is higher than that of birds in Karangwangi, Pangalengan, and Kertasari. Like area size, it is also generally accepted that there is positive correlation between the diversity of habitat and diversity of birds (Iskandar and Iskandar 2021). Our study was employed in various habitats, including upland forest, lowland forest, shrubs, plantation, riverbanks, mangrove, and rice field, while in Karangwangi was located in mixedgarden, coast, and forest; Pangelengan in the coffee agroforestry; and Kertasari was carried out in home garden, mixed-garden, and annual cash-crop garden. In addition, in terms of the local environmental conditions, Pangalengan and Kertasari are recognized as a center of vegetable farming in West Java. The intensive vegetable farming techniques along with intensive pesticides used have been occurring in Pangalengan and Kertasari of upper Citarum Watershed. Therefore, it can be presumed that the low diversity of bird species in upper Citarum Watershed areas, including Pangalengan and Kertasari, was influenced by the predominant use of intensive farming activities of vegetables, intensive use of pesticides, and rampant hunting of birds to be sold in Bandung city (Iskandar 1980, van Helvoort 1981; Hakim et al. 20020; Madani 2020).

\section{Bird diversity in Tamanjaya}

We recorded 65 species of 33 families in the Tamanjaya Village. Tamanjaya had several locations with remnant forests in hilly areas and along rocky rivers. The remnant forests in steep areas had a high diversity of plants with multiple vegetation stratifications some of which had high of 20-30 m and edible fruits, such as Bignay (Antidesma bunius). This tree attracted small fruit-eating birds, such as Loriculus pusillus and Psilopogon javensis. Psilopogon javensis was often observed in the cliffs of Gunung Cupu site and its nest was found on the roadside in Cipeucang site. Raptor species, Nisaetus bartelsii, as endemic species in Java and national symbol of Garuda Pancasila was also found in the Cipeucang and Panenjoan sites.

Remnant forests in the Cipeucang River also served as the habitat of ground snails, which are one of the preys of Hydrornis guajanus. Hydrornis guajanus may be found primarily near the water stream, especially with a forest floor covered by leaf litter (Rheindt and Eaton 2010). Phodilus badius and Otus lempiji, as nocturnal birds, were also found in this river.

\section{Bird diversity in Girimukti}

We recorded 95 species of 41 families in Girimukti Village. In general, landscapes in this village were dominated by lands that had been abandoned and became shrubs. As such, this location is a favorable habitat for several species. The raptor species, including Ictinaetus malayensis, Pernis ptilorhynchus, and Microhierax fringillarius, were only found in this area. Fruit-eating species were also recorded, such as Loriculus pusillus, Psilopogon lineatus, and Treron griseicauda. Psilopogon lineatus was only documented in this area and only once encountered in the cliff area. Shrub bird that was not found in other locations was Kittacincla malabaricus. Other species, including Gallus gallus and Hydrornis guajanus, were also not found in other areas. Hydrornis guajanus and Gallus gallus were recorded at the ground layer of the forest so that these species are categorized as terrestrial birds (Krisanti et al. 2017). 
Table 1. Bird diversity in Ciletuh-Palabuhanratu Geopark, Ciemas Subdistrict, Sukabumi District, West Java, Indonesia

\begin{tabular}{|c|c|c|c|c|c|c|c|c|c|c|}
\hline \multirow{2}{*}{ Family | Species } & \multirow{2}{*}{ Local name } & \multirow{2}{*}{ English common name } & \multicolumn{3}{|c|}{ Locations } & \multicolumn{3}{|c|}{$\begin{array}{c}\text { Conservation } \\
\text { Status }\end{array}$} & \multicolumn{2}{|c|}{ Data source } \\
\hline & & & TJ & GM & MJ & RI & IU & CI & FO & I \\
\hline \multicolumn{11}{|l|}{ Acanthizidae } \\
\hline Gerygone sulphurea (Wallace, 1864) & Manuk remetuk & Golden-bellied Gerygone & $\checkmark$ & $\checkmark$ & $\checkmark$ & & $\mathrm{LC}$ & & $\checkmark$ & \\
\hline \multicolumn{11}{|l|}{ Accipitridae } \\
\hline Haliaeetus leucogaster (Gmelin, 1788) & Heulang laut & White-bellied Sea-eagle & $\checkmark$ & $\checkmark$ & $\checkmark$ & $\checkmark$ & $\mathrm{LC}$ & II & $\checkmark$ & $\checkmark$ \\
\hline Ictinaetus malayensis (Temminck, 1822) & Heulang hideung & Black Eagle & & $\checkmark$ & & $\checkmark$ & $\mathrm{LC}$ & II & $\checkmark$ & $\checkmark$ \\
\hline Nisaetus bartelsi (Stresemann, 1924) & Elang Jawa & Javan Hawk-eagle & $\checkmark$ & & & $\checkmark$ & EN & II & $\checkmark$ & \\
\hline Pernis ptilorhynchus (Temminck, 1821) & Elang & Oriental Honey-buzzard & & $\checkmark$ & & $\checkmark$ & $\mathrm{LC}$ & II & $\checkmark$ & \\
\hline Spilornis cheela (Latham, 1790) & Heulang ruyuk & Crested Serpent Eagle & $\checkmark$ & $\checkmark$ & & $\checkmark$ & $\mathrm{LC}$ & II & $\checkmark$ & \\
\hline Spizaetus cirrhatus (Gmelin, 1788) & Heulang brontok & Crested Hawk-Eagle & $\checkmark$ & $\checkmark$ & $\checkmark$ & $\checkmark$ & LC & II & $\checkmark$ & \\
\hline \multicolumn{11}{|l|}{ Aegithinidae } \\
\hline Aegithina tiphia (Linnaeus, 1758) & Cipeuw & Common Iora & $\checkmark$ & $\checkmark$ & $\checkmark$ & & $\mathrm{LC}$ & & $\checkmark$ & $\checkmark$ \\
\hline \multicolumn{11}{|l|}{ Alcedinidae } \\
\hline Alcedo coerulescens (Vielliot, 1818) & Burung udang & Cerulean Kingfisher & & & $\checkmark$ & & $\mathrm{LC}$ & & $\checkmark$ & \\
\hline Alcedo meninting (Horsfield, 1821) & Manuk hurang & Blue-eared Kingfisher & & & $\checkmark$ & & $\mathrm{LC}$ & & $\checkmark$ & \\
\hline Halcyon cyanoventris (Vieillot, 1818) ${ }^{2}$ & Cekahkeh & Javan Kingfisher & $\checkmark$ & $\checkmark$ & $\checkmark$ & & $\mathrm{LC}$ & & $\checkmark$ & $\checkmark$ \\
\hline Halcyon smyrnensis (Linnaeus, 1758) & Manuk hurang & White-breasted Kingfisher & & $\checkmark$ & & & $\mathrm{LC}$ & & & $\checkmark$ \\
\hline Todiramphus chloris (Boddaert, 1783) & Cekakak & Collared Kingfisher & $\checkmark$ & $\checkmark$ & $\checkmark$ & & $\mathrm{LC}$ & & $\checkmark$ & $\checkmark$ \\
\hline Todiramphus sanctus (Vigors \& Horsfeld, 1827) & Cekakak & Sacred Kingfisher & & & $\checkmark$ & & LC & & $\checkmark$ & \\
\hline \multicolumn{11}{|l|}{ Apodidae } \\
\hline Apus affinis (Gray, 1830) & Kapinis rumah & Little Swift & $\checkmark$ & & & & $\mathrm{LC}$ & & $\checkmark$ & \\
\hline Collocalia linchi (Horsfield \& Moore, 1854) & Walet & Linchi Swiftlet & $\checkmark$ & $\checkmark$ & $\checkmark$ & & $\mathrm{LC}$ & & $\checkmark$ & $\checkmark$ \\
\hline \multicolumn{11}{|l|}{ Ardeidae } \\
\hline Ardea purpurea (Linnaeus, 1766) & Kokondangan & Purple Heron & & $\checkmark$ & & & $\mathrm{LC}$ & & & $\checkmark$ \\
\hline Butorides striata (Linnaeus, 1758) & Goak & Green-backed Heron & & & $\checkmark$ & & $\mathrm{LC}$ & & $\checkmark$ & \\
\hline Egretta sacra (Gmelin, 1789) & Kuntul karang & Pasific Reef-egret & & & $\checkmark$ & & $\mathrm{LC}$ & & $\checkmark$ & \\
\hline \multicolumn{11}{|l|}{ Bucerotidae } \\
\hline Rhyticeros undulatus (Shaw, 1811) & Julang & Rhinoceros Hornbill & & $\checkmark$ & & $\checkmark$ & VU & II & & $\checkmark$ \\
\hline Buceros rhinoceros (Linnaeus, 1758) & Rangkong & Wreathed Hornbill & & $\checkmark$ & & $\checkmark$ & VU & II & & $\checkmark$ \\
\hline \multicolumn{11}{|l|}{$\begin{array}{l}\text { Campephagidae } \\
\text { Cas (t) }\end{array}$} \\
\hline Lalage nigra (Forster, 1781) & Manuk muncang & Pied Triller & $\checkmark$ & & $\checkmark$ & & $\mathrm{LC}$ & & $\checkmark$ & \\
\hline \multicolumn{11}{|l|}{ Caprimulgidae } \\
\hline Caprimulgus affinis (Horsfield, 1821) & Cuhcur & Savanna Nightjar & & $\checkmark$ & & & $\mathrm{LC}$ & & & $\checkmark$ \\
\hline \multicolumn{11}{|l|}{ Chloropseidae } \\
\hline Chloropsis sonnerati (Jardine \& Selby, 1827) & Manuk daun & Greater Green Leafbird & & $\checkmark$ & & $\checkmark$ & VU & & & $\checkmark$ \\
\hline \multicolumn{11}{|l|}{ Charadriidae } \\
\hline Pluvialis squatarola (Linnaeus, 1758) & Belibis laut & Grey Plover & & $\checkmark$ & & & $\mathrm{LC}$ & & & $\checkmark$ \\
\hline
\end{tabular}




\section{Cisticolidae}

Orthotomus ruficeps (Lesson, 1830)

Orthotomus sepium (Horsfield, 1821)

Orthotomus sutorius (Pennant, 1769)

Prinia flaviventris (Delessert, 1840)

Prinia polychroa (Temminck, 1828)

\section{Columbidae}

Chalcophaps indica (Linnaeus, 1758)

Ducula bicolor (Scopoli, 1786)

Geopelia striata (Linnaeus, 1766)

Macropygia emiliana (Bonaparte, 1854)

Streptopelia chinensis (Scopoli, 1786)

Streptopelia bitorquata (Temminck, 1809)

Treron capellei (Temminck, 1823)

Treron griseicauda (Wallace, 1863)

Treron vernans (Linnaeus, 1771)

\section{Coriciidae}

Eurystomus orientalis (Linnaeus, 1766)

Corvidae

Corvus enca (Horsfield, 1822)

Crypsirina temia (Daudin, 1800)

Cuculidae

Cacomantis merulinus (Scopoli, 1786)

Cacomantis sepulcralis (Vigors \& Horsfield, 1826)

Cacomantis sonneratii (Latham, 1790)

Centropus bengalensis (Gmelin, 1788)

Centropus nigrorufus (Cuvier, 1817)

Centropus sinensis (Stephens, 1815)

Phaenicophaeus curvirostris (Shaw, 1810)

Surniculus lugubris (Horsfield, 1821)

Dicaeidae

Dicaeum chrysorrheum (Temminck \& Laugier, 1829) Dicaeum trigonostigma (Scopoli, 1786)

Dicaeum trochileum (Sparrman, 1789)

\section{Dicruridae}

Dicrurus macrocercus (Vieillot, 1817)

Dicrurus paradiseus (Linnaeus, 1766)

\section{Estrildidae}

Lonchura leucogastroides (Horsfield \& Moore, 1856) Lonchura maja (Linnaeus, 1766)

Lonchura punctulata (Linnaeus, 1758)

Padda oryzivora (Linnaeus, 1758)

\section{Falconidae}

Falco moluccensis (Bonaparte, 1850)

Microhierax fringillarius (Drapiez, 1824)
Esel nangka

Esel nangka

Nangkaan

Pacikrak

Pacikrak

Limbukan

Pergam laut

Perkutut

Manuk uncal

Tikukur

Manuk puter

Punai

Punai

Walik

Tiong

Gaak

Saeran leuweung

Sirit uncuing

Sirit uncuing

Sirit uncuing

Dudut

Bubut

Dudut

Londrok

Aeh aeh

Manuk cabe

Ceced

Manuk seupah

Saeran

Manuk rambai

Manuk piit

Bondol

Peking

Galatik

Dedalik, Alap-alap Alap-alap
Ashy Tailorbird

Olive-backed Tailorbird

Common Tailorbird

Yellow-bellied Prinia

Brown Prinia

Emerald Dove

Pied Imperial-pigeon

Zebra Dove

Ruddy Cuckoo-dove

Spotted Dove

Sunda Collared-dove

Large Green-pigeon

Grey-cheecked Green-pigeon

Pink-necked Green-pigeon

Oriental Dollarbird

Slender-billed Crow

Racket-tailed Treepie

Plaintive Cuckoo

Brush Cuckoo

Banded Bay Cuckoo

Lesser Coucal

Javan Coucal

Greater Couca

Chestnut-breasted Malkoha

Square-tailed Drongo-cuckoo

Yellow-vented Flowerpecker

Orange-bellied Flowerpecker

Scarlet-headed Flowerpecker

Black Drongo

Greater Racquet-tailed Drongo

Javan Munia

White-headed Munia

Scaly-breasted Munia

Java Sparrow

Spotted Kestrel

Black-thighed Falconet

t

$\begin{array}{llllll} & \checkmark & & \text { LC } & & \checkmark \\ \checkmark & & & \text { LC } & \checkmark & \\ \checkmark & \checkmark & \checkmark & \text { LC } & \checkmark & \checkmark \\ \checkmark & & & \text { LC } & \checkmark & \\ \checkmark & \checkmark & \text { LC } & \checkmark & \checkmark \\ & & & & & \\ & \checkmark & & \text { LC } & \checkmark & \checkmark \\ & \checkmark & & \text { LC } & \checkmark & \\ & \checkmark & \checkmark & \text { LC } & \checkmark & \checkmark \\ & \checkmark & & \text { LC } & & \checkmark \\ & \checkmark & \checkmark & \text { LC } & \checkmark & \checkmark \\ & \checkmark & & \text { LC } & & \checkmark \\ \checkmark & \checkmark & & \text { VU } & & \checkmark \\ & \checkmark & \text { LC } & \checkmark & \checkmark \\ & \checkmark & \text { LC } & & \checkmark\end{array}$

$\checkmark$ LC

$\begin{array}{lllll} & \checkmark & & & \text { LC } \\ \checkmark & \checkmark & \checkmark & \checkmark & \text { LC }\end{array}$

$\checkmark-\checkmark$ LC

$\checkmark \quad \checkmark \quad \checkmark$ LC

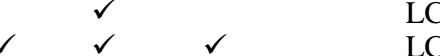

$\checkmark \quad$ VU

LC 
Hemiprocnidae

Hemiprocne longipennis (Rafinesque, 1802)

Luluis

Kapinis

Kapinis

(Girundo tahitica (Gmelin, 1789)

Laniidae

Lanius schach (Linnaeus, 1758)

Locustellidae

Megalurus palustris (Horsfield, 1821)

\section{Megalaimidae}

Psilopogon australis (Horsfield, 1821)

Psilopogon armillaris (Temminck, 1821)

Psilopogon javensis (Horsfield, 1821)

Psilopogon lineatus (Vieillot, 1816)

\section{Motacillidae}

Anthus novaeseelandiae (Gmelin, 1789)

\section{Muscicapidae}

Kittacincla malabarica (Scopoli, 1788)

Copsychus saularis (Linnaeus, 1758)

Cyornis rufigastra (Raffles, 1822)

Enicurus leschenaulti (Vieillot, 1818)

Enicurus velatus (Temminck, 1822)

Nectariniidae

Anthreptes malacensis (Scopoli, 1786)

Arachnothera longirostra (Latham, 1790)

Arachnothera robusta (Muller \& Schlegel, 1845)

Nectarinia calcostetha (Jardine, 1843)

Nectarinia jugularis (Linnaeus, 1766)

Orilidae

Oriolus chinensis (Linnaeus, 1766)

Paridae

Parus major (Linnaeus, 1758)

Passeridae

Passer montanus (Linnaeus, 1758)

Pellorneidae

Malacocincla sepiaria (Horsfield, 1821)

Malacopteron cinereum (Eyton, 1839)

Pellorneum capistratum (Temminck, 1823)

Phasianidae

Gallus gallus (Linnaeus, 1758)

Gallus varius (Shaw, 1798)

Coturnix chinensis (Linnaeus, 1766)

\section{Picidae}

Dendrocopos macei (Vieillot, 1818)

Dendrocopos moluccensis (Gmelin, 1788)
Toed

Tektekreyod

Ungkut-ungkut gunung

Tohtor

Tulung tumpuk

Bultok

Apung

Peteng

Manuk haur

Sikatan bakau

Manintin

Manintin

Cuit

Kelaces, Manuk jantung

Kelaces

Cuit

Cuit

Bincarung

Gelatik batu

Golejra

Kancilan

Asi Topi-sisik

Cica bata

Kasintu

Cangehgar

Puyuh

Caladi kotok, Caladi muncang Caladi tikotok
Grey-rumped Treeswift

Barn Swallow

Tahiti Swallow

Long-tailed Shrike

Striated Grassbird

Yellow-eared Barbet

Flame-fronted Barbet

Black-banded Barbet

Lineated Barbet

Australasian Pipit

White-rumped Shama

Oriental Magpie-robin

Mangrove Blue-flycatcher

White-crowned Forktail

Sunda Forktail

Brown-throated Sunbird

Little Spiderhunter

Long-billed Spiderhunter

Copper-throated Sunbird

Olive-backed Sunbird

Black-naped Oriole

Great Tit

Eurasian Tree Sparrow

Horsfield's Babbler

Scaly-crowned Babbler

Rufous-browed Babbler

Red Junglefowl

Green Junglefowl

King Quail

Fulvous-breasted Woodpecker Sunda Pygmy Woodpecker 


\section{Pittidae}

Hydrornis guajanus (Müller, 1776)

Ploceidae

Ploceus manyar (Horsfield, 1821)

Psittacidae

Loriculus pusillus (Gray, 1859) ${ }^{2}$

Psittacula alexandri (Linnaeus, 1758)

Pycnonotidae

Alophoixus bres (Lesson, 1832)

Alophoixus ochraceus (Moore, 1854)

Brachypodius atriceps (Temminck, 1822)

Pycnonotus aurigaster (Vieillot, 1818)

Pycnonotus goiavier (Scopoli, 1786)

Pycnonotus melanicterus (Gmelin, 1789)

Pycnonotus plumosus (Blyth, 1845)

Rallidae

Amaurornis phoenicurus (Pennant, 1769)

Lewinia striata (Linnaeus, 1766)

\section{Rhipiduridae}

Rhipidura javanica (Sparrman, 1788)

Scolopacidae

Tringa hypoleucos (Linnaeus, 1758)

Sittidae

Sitta frontalis (Swainson, 1820)

Strigidae

Ketupa ketupu (Horsfield, 1821)

Otus lempiji (Horsfield, 1821)

Sturnidae

Acridotheres javanicus (Cabanis, 1851) ${ }^{2}$

Gracupica contra (Linnaeus, 1758)

Turdidae

Geokichla citrina (Latham, 1790)

Geokichla interpres (Temminck, 1826)

Zoothera andromedae (Temminck, 1826)

Turnicidae

Turnix suscitator (Gmelin, 1789)

Tytonida

Phodilus badius (Horsfield, 1821)

Tyto alba (Scopoli, 1769)

\section{Zosterophidae}

Lopozosterops javanicus (Horsfield, 1821)

Zosterops palpebrosus (Temminck, 1824)

Manuk kacamata
Paok

Manyar

Serindit

Ekek

Kores

kutilang jenggot

Kuricang

Cangkurileung

Jogjog

Trenggoleng

Corok-corok

Kareo

Mandar

Manuk sapu

Tili

Saleser

Kutuk beluk

Bueuk

Kerak kerbau

Kerak omas

Manuk cacing

Anis kembang

Manuk cacing

Gemak Loreng

Serak bukit

Koreak
Javan Banded Hydrornis

Streaked Weaver

Yellow-throated Parrot Red-breasted Parakeet

Brown-cheeked Bulbul

Ochraceous Bulbul

Black-headed Bulbul

Sooty-headed Bulbul

Yellow-vented Bulbul

Black-capped Bulbul

Olive-winged Bulbul

White-breasted Waterhen

Slaty-breasted Rail

Sunda Pied Fantail

Common Sandpiper

Velvet-fronted Nuthatch

Buffy Fish-owl

Sunda Scops-owl

Javan Myna

Asian Pied Starling

Orange-headed Thrush

Chestnut-capped Thrush

Sunda Thrush

Barred Buttonquai

Oriental Bay-owl

Common Barn-ow

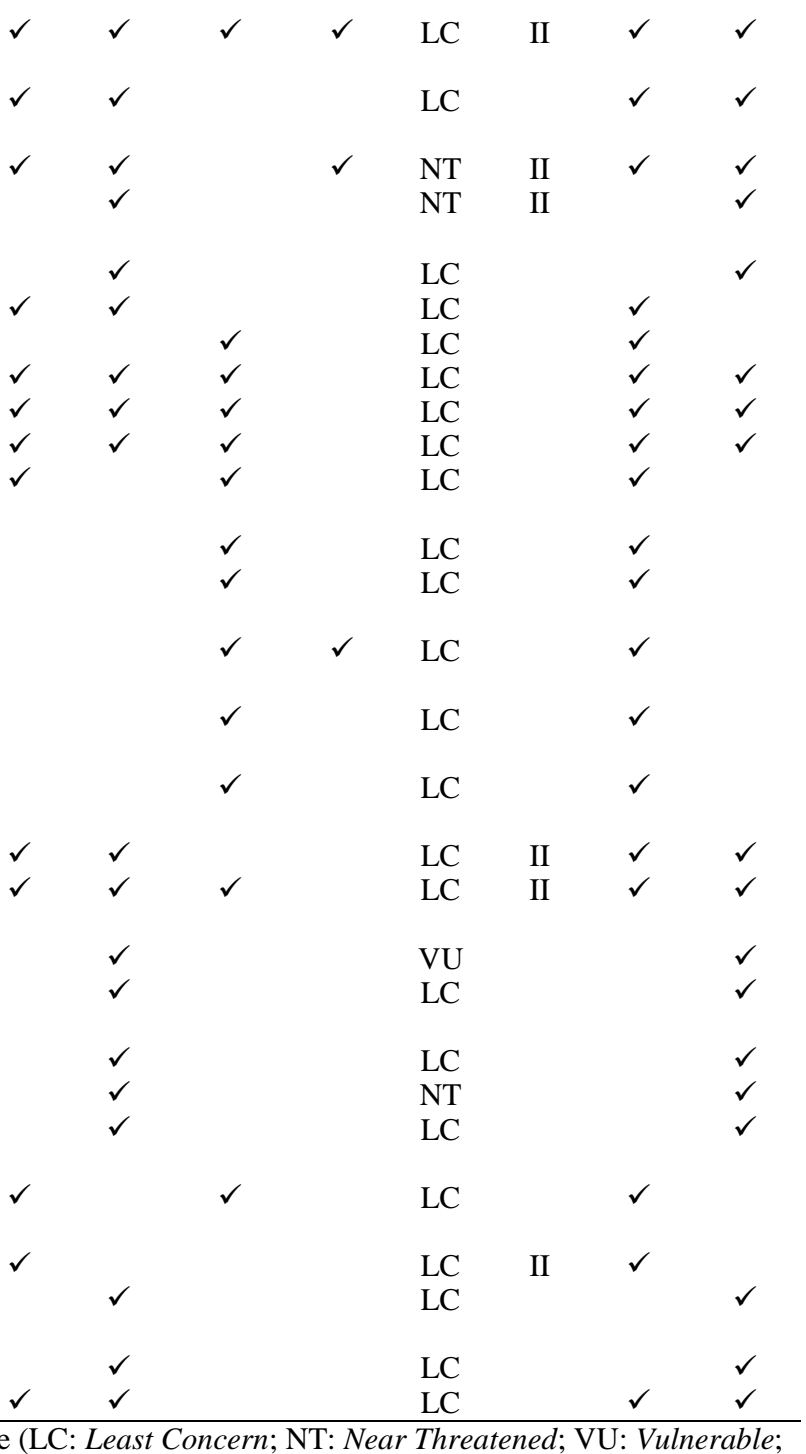

EN: Endangered); CI: Convention on International Trade in Endangered Species: FO: Field observation; I:Interviews. ${ }^{1}$ Endemic to the Java Island; ${ }^{2}$ Endemic to the Java-Bali Island; ${ }^{3}$ Migratory Bird. Sources: Primary Data (2016-2017); IUCN (2021); UNEP WCMC (Comps) 2021; and Regulation of the Minister of Environment and Forestry of the Republic of Indonesia No. P 106/2018 on Protected Plant and Animal Species. 
Table 2. Bird species are recognized by village communities and folk classification of birds based on local knowledge

\begin{tabular}{|c|c|c|c|c|c|c|}
\hline Voice & Color & Body size & Habitat and nest & Habit & Morphology & Feeding Guild \\
\hline Ketupa ketupu (Horsfield, 1821) & $\begin{array}{l}\text { Haliaeetus leucogaster } \\
\text { (Gmelin, 1788) }\end{array}$ & $\begin{array}{l}\text { Dendrocopos } \\
\text { moluccensis (Gmelin, } \\
1788 \text { ) }\end{array}$ & $\begin{array}{l}\text { Rhyticeros undulatus } \\
\text { (Shaw, 1811) }\end{array}$ & $\begin{array}{l}\text { Acridotheres javanicus } \\
\text { (Cabanis, 1851) }\end{array}$ & $\begin{array}{l}\text { Zosterops palpebrosus } \\
\text { (Temminck, 1824) }\end{array}$ & $\begin{array}{l}\text { Treron griseicauda } \\
\text { (Wallace, } 1863 \text { ) }\end{array}$ \\
\hline Pycnonotus aurigaster (Vieillot, 1818) & $\begin{array}{l}\text { Ardea purpurea } \\
\text { (Linnaeus, 1766) }\end{array}$ & $\begin{array}{l}\text { Parus major (Linnaeus, } \\
1758 \text { ) }\end{array}$ & $\begin{array}{l}\text { Buceros rhinoceros } \\
\text { (Linnaeus, 1758) }\end{array}$ & $\begin{array}{l}\text { Cacomantis sepulcralis } \\
\text { (Vigors \& Horsfield, } \\
\text { 1826) }\end{array}$ & $\begin{array}{l}\text { Dicrurus paradiseus } \\
\text { (Linnaeus, 1766) }\end{array}$ & $\begin{array}{l}\text { Arachnothera robusta } \\
\text { (Muller \& Schlegel, } \\
\text { 1845) }\end{array}$ \\
\hline $\begin{array}{l}\text { Lonchura leucogastroides (Horsfield \& } \\
\text { Moore, 1856) }\end{array}$ & $\begin{array}{l}\text { Dendrocopos macei } \\
\text { (Vieillot, 1818) }\end{array}$ & $\begin{array}{l}\text { Coturnix chinensis } \\
\text { (Linnaeus, 1766) }\end{array}$ & $\begin{array}{l}\text { Pluvialis squatarola } \\
\text { (Linnaeus, 1758) }\end{array}$ & $\begin{array}{l}\text { Nectarinia/Cinnyris } \\
\text { jugularis (Linnaeus, 1766) }\end{array}$ & $\begin{array}{l}\text { Dicrurus macrocercus } \\
\text { (Vieillot, 1817) }\end{array}$ & \\
\hline Prinia polychroa $($ Temminck, 1828$)$ & $\begin{array}{l}\text { Treron vernans (Linnaeus, } \\
1771 \text { ) }\end{array}$ & $\begin{array}{l}\text { Macropygia emiliana } \\
\text { (Bonaparte, 1854) }\end{array}$ & $\begin{array}{l}\text { Enicurus velatus } \\
\text { (Temminck, 1822) }\end{array}$ & $\begin{array}{l}\text { Malacopteron cinereum } \\
\text { (Eyton, 1839) }\end{array}$ & $\begin{array}{l}\text { Alophoixus bres (Lesson, } \\
1832 \text { ) }\end{array}$ & \\
\hline Orthotomus ruficeps (Lesson, 1830) & $\begin{array}{l}\text { Padda oryzivora } \\
\text { (Linnaeus, } 1758)\end{array}$ & $\begin{array}{l}\text { Treron capellei } \\
\text { (Temminck, 1823) }\end{array}$ & $\begin{array}{l}\text { Enicurus leschenaulti } \\
\text { (Vieillot, 1818) }\end{array}$ & & & \\
\hline Caprimulgus affinis (Horsfield, 1821) & $\begin{array}{l}\text { Chloropsis sonnerati } \\
\text { (Jardine \& Selby, 1827) }\end{array}$ & $\begin{array}{l}\text { Centropus sinensis } \\
\text { (Stephens, 1815) }\end{array}$ & $\begin{array}{l}\text { Ploceus manyar } \\
\text { (Horsfield, 1821) }\end{array}$ & & & \\
\hline Tyto alba (Scopoli, 1769) & $\begin{array}{l}\text { Geopelia striata } \\
\text { (Linnaeus, } 1766 \text { ) }\end{array}$ & & $\begin{array}{l}\text { Sitta frontalis } \\
\text { (Swainson, 1820) }\end{array}$ & & & \\
\hline Aegithina tiphia (Linnaeus, 1758) & $\begin{array}{l}\text { Pycnonotus melanicterus } \\
\text { (Gmelin, 1789) }\end{array}$ & & & & & \\
\hline Orthotomus sutorius (Pennant, 1769) & $\begin{array}{l}\text { Hydrornis guajanus } \\
\text { (Müller, 1776) }\end{array}$ & & & & & \\
\hline Lanius schach (Linnaeus, 1758) & $\begin{array}{l}\text { Dicaeum trochileum } \\
\text { (Sparrman, 1789) }\end{array}$ & & & & & \\
\hline Corvus enca (Horsfield, 1822) & $\begin{array}{l}\text { Oriolus chinensis } \\
\text { (Linnaeus, 1766) }\end{array}$ & & & & & \\
\hline \multicolumn{7}{|l|}{ Pycnonotus goiavier (Scopoli, 1786) } \\
\hline \multicolumn{7}{|l|}{ Gallus varius (Shaw, 1798) } \\
\hline \multicolumn{7}{|l|}{ Streptopelia chinensis (Scopoli, 1786) } \\
\hline \multicolumn{7}{|l|}{ Centropus bengalensis (Gmelin, 1788) } \\
\hline \multicolumn{7}{|l|}{ Psilopogon javensis (Horsfield, 1821) } \\
\hline \multicolumn{7}{|l|}{ Psilopogon haemacephalus (Müller, 1776) } \\
\hline \multicolumn{7}{|l|}{ Psilopogon lineatus (Vieillot, 1816) } \\
\hline \multicolumn{7}{|l|}{ Todiramphus chloris (Boddaert, 1783) } \\
\hline \multicolumn{7}{|l|}{ Halcyon cyanoventris (Vieillot, 1818) } \\
\hline \multicolumn{7}{|l|}{ Phaenicophaeus curvirostris (Shaw, 1810) } \\
\hline \multicolumn{7}{|l|}{ Streptopelia bitorquata (Temminck, 1809) } \\
\hline Ictinaetus malayensis (Temminck, 1822) & & & & & & \\
\hline
\end{tabular}




\section{Bird diversity in Mandrajaya}

We recorded 58 species of 31 family in Mandrajaya Village. In general, land cover in Mandrajaya consisted of rice fields, plantations, mangroves, and lowland forests. The bird species found in this location were quite diverse with some species were can only be found in this location (e.g. Actitis hypoleucos and Lewinia striata) because the distinct habitats including coastal area and mangrove forests. In addition, a raptor species Haliaeetus leucogaster was only found in Mandrajaya. In Mandrajaya, there is Cibanteng Nature Reserve which has natural forest land cover. Bird species recorded here included lowland forestdwelling birds, such as Pycnonotus plumosus, Sitta frontalis, and Brachypodius atriceps. Migrant bird, namely Tringa hypoleucos, was recorded along the coast and mangrove forests in Mandrajaya. Tringa hypoleucos is normally breeding in Africa and Eurasia during summer, and in winter this bird usually migrates to southern hemisphere up to Australia (Mackinon et al. 2010).

Local knowledge on bird species and bird classification

Based on the local knowledge, the respondents recognized 79 species in the study area. Among these, 19 species were considered as rare species, such as Peteng (Kittacincla malabaricus), Kokondangan (Ardea purpurea), Bincarung (Oriolus chinensis), and Cuhcur (Caprimulgus affinis) (Table 3). Besides, eight species were considered extinct or never seen again in the forest and village, including Manuk cacing (Zoothera andromedae), Manuk cacing (Geokichla interpres), Anis (Geokichla citrina), Manuk haur (Copsychus saularis), Julang (Rhinoceros undulatus), Rangkong (Rhyticeros rhinoceros), Gaak (Corvus enca), and Cangehgar (Gallus varius). Of 19 species considered rare based on the respondents, 11 species were still found through field observation. Birds that were considered extinct based on respondents but still found through field observation were only Cangehgar (Gallus varius). Further studies are needed to ensure bird presence and the impact of loss or extinction of birds in the Ciletuh-Palabuhanratu Geopark.

Folk classification refers to how the local community naming and categorizing birds. Based on the local people in the Ciletuh-Palabuhanratu Geopark, birds can be divided into eight classifications, namely based on distinctive voice/vocalization, special color of feather, body size, habitat and nest, distinctive behavior/habit, morphology, and feeding guild (Tables 2 and 4). In daily lives, the local people often hear distinctive chirping birds, so people give the name according to its distinctive voice. For example, Ketupa ketupu known as Kutuk beluk because this bird has special voice which sounds like "kutuk beluk-kutuk belukkutuk beluk" at night. According to the morphology classification, the village community gives the bird's name based on tail form and other characteristics. For example, Zosterops palpebrosus is knowns as Manuk Kacamata because it has a white circle around its eyes like glasses.

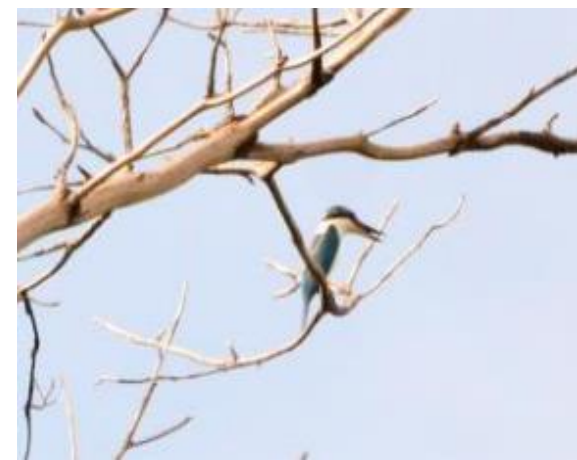

A

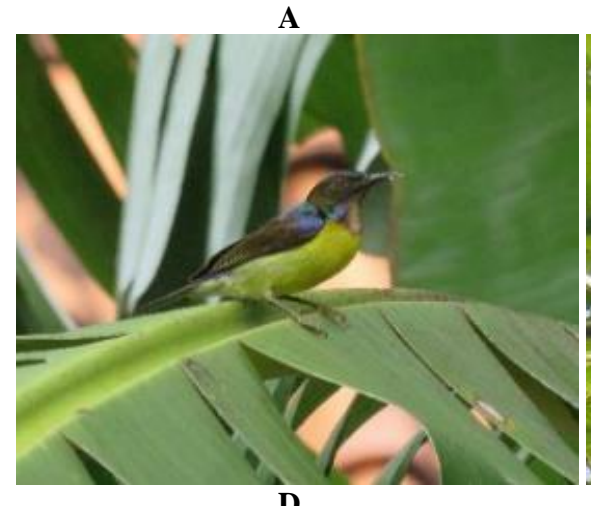

D

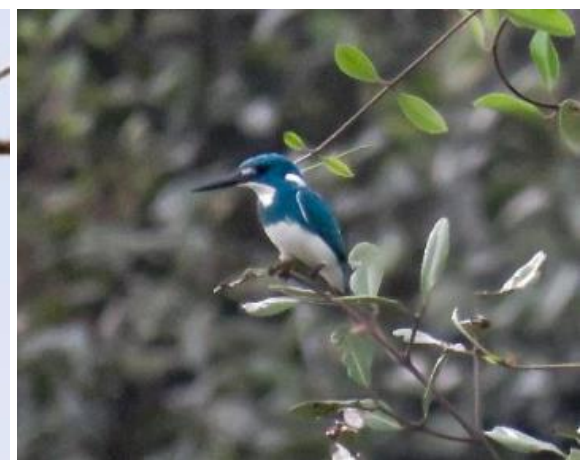

B

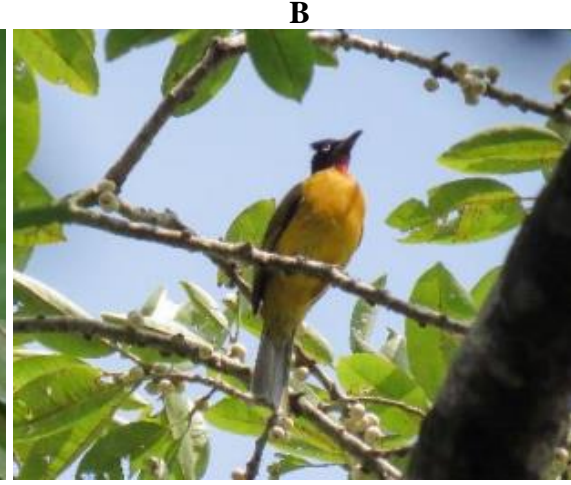

$\mathbf{E}$

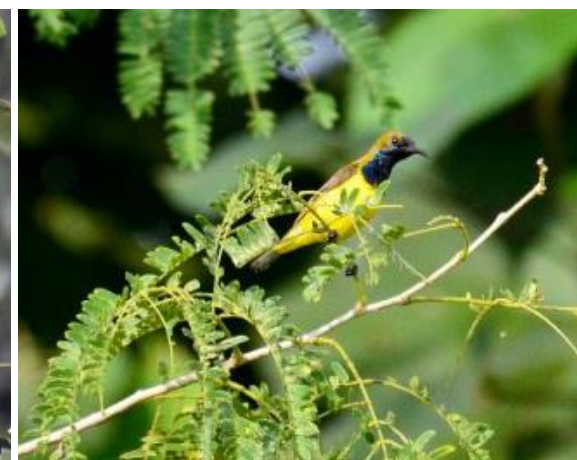

C

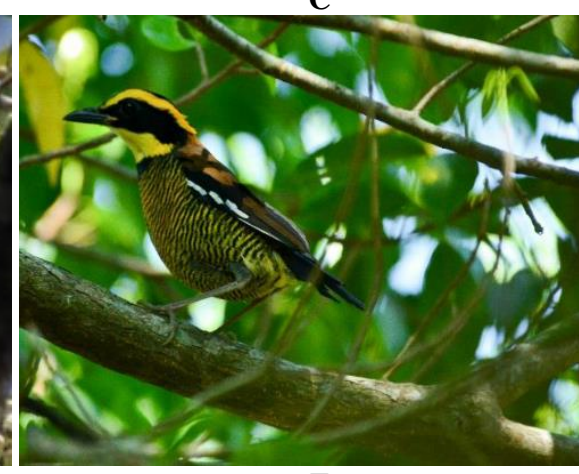

$\mathbf{F}$

Figure 2. Some birds recorded in the Amphitheater area, Ciletuh-Palabuhanratu Geopark, Sukabumi: A. Todirhamphus chloris, B. Alcedo coerulescens, C. Cinnyris/Nectarinia jugularis, D. Anthreptes malacensis, E. Pycnonotus melanicters, F. Hydrornis guajanus. Photo by syahrasf 
Table 3. Birds considered as rare species in Ciletuh-Palabuhanratu Geoparpark based on the respondents' perception

\begin{tabular}{llll}
\hline \multicolumn{1}{c}{ Local name } & \multicolumn{1}{c}{ Indonesian name } & \multicolumn{1}{c}{ English common name } & \multicolumn{1}{c}{ Scientific name } \\
\hline Belibis laut & Cerek besar & Grey plover & Puvialis squatarola Linnaeus, 1758 \\
Bincarung & Kepudang kuduk-hitam & Black-naped oriole & Oriolus chinensis (Linnaeus, 1766) \\
Cuhcur & Cabak kota & Savannah nightjar & Caprimulgus affinis (Horsfield, 1821) \\
Ekek & Betet biasa & Red-breasted parakeet & Psittacula alexandri (Linnaeus, 1758) \\
Golejra & Burung gereja-erasia & Eurasian tree sparrow & Passer montanus (Linnaeus, 1758) \\
Kasintu & Ayam hutan-merah & Red junglefowl & Gallus gallus (Linnaeus, 1758) \\
Kelatik kapas, Galatik & Gelatik jawa & Java sparrow & Padda oryzivora (Linnaeus, 1758) \\
Kerak omas & Jalak suren & Asian pied starling & Gracupica contra (Linnaeus, 1758) \\
Kokondangan & Cangak merah & Purple heron & Ardea purpurea (Linnaeus, 1766) \\
Kores & Empuloh janggut & Grey-cheeked bulbu & Alophoixus bres (Lesson, 1832) \\
Limukeun, Limbukan & Delimukan zamrud & Emerald dove & Chalcophaps indica (Linnaeus, 1758) \\
Londrok & Kadalan birah & Chestnut-breasted malkoha & Phaenicophaeus curvirostris (Shaw, 1810) \\
Luluis & Tepekong jambul & Grey-rumped treeswift & Hemiprocne longipennis (Rafinesque, 1802) \\
Manuk hurang & Cekakak belukar & White-throated kingfisher & Halcyon smyrnensis (Linnaeus, 1758) \\
Manuk rambai & Srigunting batu & Greater racket-tailed & Dicrurus paradiseus (Linnaeus, 1766) \\
Paok & Paok pancawarna & Banded pitta & Hydronis guajanus (Müller, 1776) \\
Peteng, Murai batu & Kucica hutan & Whited-rumped shama & Kittacincla malabarica (Scopoli, 1788) \\
Saeran gunting & Srigunting hitam & Black drongo & Dicrurus macrocerceus (Vieillot, 1817) \\
Tukang kewiwi, Bueuk & Celepuk reban & Collared scopsowl & Otus lempiji (Horsfield, 1821) \\
\hline
\end{tabular}

Some birds are known to have special distinctive vernacular name due to specific feather colors. For example, Ardea purpurea is commonly known by local village people as Kokondangan because of its reddishbrown color (In Sundanese means "Kondang"). Based on the body size, the community mentions small birds as Muncang, which is in Sundanese it means tiny seeds like candlenut. For example, Dendrocopos moluccensis is known as Caladi muncang. According to the habits, people often see the bird's habits in their daily lives. For instance, Acridotheres javanicus is known as Kerak kebo ("kebo" in Sundanese means buffalo). Thus, since the Acridotheres javanicus has been predominantly seen perching on the buffalo's back to find various insects in the paddy field, this bird is locally named Kerak kebo. Meanwhile, bird of Sitta frontalis is locally called as Manuk salésér (in Sundanese, "salésér" means creeping). Because this bird has distinctive behavior usually creeping on the trunk or branch of tree.

Some vernacular names of birds are known due to characteristics of their feed. For instance, Treron giseicauda is locally known as Walik harendong because this bird is frequently eats Harendong (Melastoma malabatricum) fruits. People recognize birds based on their habitat, such as Enicurus leschenaulti which is known as Manintin that is traditionally classified as bird of a river water habitat, because this bird was frequently seen playing in a water stream of the river.

As can be seen from the Table 2 and Table 3 that the folk classification of birds based on local knowledge of the people of the Ciletuh-Palabuhanratu-Geoparpark is rather similar to Karangawangi Village people in that birds are categorized based on distinctive voice/vocalization, special color, distinctive behavior/habit, special habitat and nest, morphological characteristics, and special role in village ecosystem (Iskandar et al. 2016). This is because both local village community of the Ciletuh-Palabuanratu Geopark in Sukabumi and the village community of the Karangwangi, Cianjur, have similar cultural backgrounds of Sundanese culture. Culturally, based on respondents, they obtain local knowledge on birds from various sources, namely parents, personal experiences, and friends (Table 4). Therefore, Pam (2017) may be correct when she says that three methods of the local knowledge transmission of birds within the cultural transmission framework, namely vertical, horizontal and oblique transmission. Vertical transmission refers to learning which occurs from older people into younger generation, while horizontal transmission refers to transfer of information or knowledge between peers (friends). On other hand, oblique transmission refers to the intergenerational knowledge transfer which takes place between non-related members of the community. Similarly, according to Puri (1997), the local knowledge is culturally transmitted into three methods; parental learning, peer group learning, and individual learning.

\section{Local knowledge on bird functions and utilizations}

Based on the interviews with informants, in most cases, the village people in Ciletuh-Palabuhanratu Geopark have profound knowledge on bird functions. They recognize that various birds in village ecosystem have some ecological, socio-cultural, and socio-economic functions. In terms of ecological functions, some bird species including eagles are known for pest control in rice fields and swidden farming. Eagles, such as Spilornis cheela, are very helpful for the village farmers in controlling rats in the rice field because this bird usually eats rats. Some forest-dwelling birds, including Rhyticeros undulatus and Buceros rhinoceros are known as seed dispersers. Since these birds commonly eat forest tree fruits, the seeds of the fruit are distributed and may germinate in the forest. Some birds, however, are considered harmful to farmers, because they eat paddy, including Passer montanus, Lonchura punctulata, and Psittacula alexandri are known as rice pests (hama pare) in the rice field as well as in swidden farming. 
In addition, the village people in Ciletuh-Palabuhanratu Geoparpark recognize the ecological function of bird as a sign of season changes. For example, piit (Lonchura leucogastroides) and manyar (Ploceus manyar) are considered indicators of changing seasons. The presence of piit crowds in rice fields and swidden cultivation fields can be traditionally used by villagers as an indicator that the rainy season has come. Additionally, the presence of Manyar in the village indicates that the dry season is coming which is indicated by Manyar nesting in the trees which grow near the rice field. The villagers' knowledge on the prediction of season is similar to that practiced in the past by Javanese farmers. In the past, the Manyar bird was used to predict season which was called the Pranata Mangsa. For example, when manyar is hatching and feeding the cheaper because of a lot of paddy seeds, it indicates Mangsa Dhesta, which lasts from April $10^{\text {th }}$ until May $11^{\text {th }}$, and the dry season will come soon (Rimanang 2016).

In terms of cultural functions, several bird species are known to have social and cultural values. For instance, Ictinaetus malayensis is known as the folklore legend on heulang kelik, Toed (Lanius schach) is traditionally consumed by child so that the child is good at talking like a Toed bird that chirps. Meanwhile, if the villagers hear the Uncuing bird (Cacomantis sepulcralis) constantly in a hamlet, it is considered by the village people that it is a sign that someone will die in that hamlet.

In the past, the village people had some myths about birds. For example, some birds are considered to bring bad luck or disaster to people who hunt, kill, keeping birds, or disturb certain birds. Those species referred to Uncuing (Cacomantis sepulcralis), Hingkik (Tyto alba), Lok-lok (unidentified night bird species), Bueuk (Ketupa ketupu), and Gaak (Corvus enca). Because these birds have been considered a myth and traditionally protected by the village people, the population of these birds are higher. Nowadays, however, many village people do not believe anymore in the myths of birds. The consequence of the decrease or loss of myths on birds may cause many people to hunt birds, including sacred birds as told in the myths in the past.

Nowadays, many people of both the villagers themselves and outsiders have predominantly hunt birds in Girimukti and Mandajaya of the Ciletuh-Palabuhanratu Geopark. Some traditional and modern methods, including pitapak/ jiret/ racik, jaring, pitangkeb, leugeut, and bedil are commonly used by bird hunters to hunt birds. Pitapak is a hunting method that uses rachis of sugar palm (harupat) or bamboo to trap birds, including Streptopelia chinensis, Geopelia striata, Hydrornis guajanus, Gallus gallus, and Coturnix chinensis. Racik is a hunting method that uses nylon to catch birds, particularly Treron griseicauda and Treron vernans. Similar to the fishing, Racik method uses bait, e.g., Ficus fruits. When a bird walks and steps on the nylon mesh and eats the bait, the nylon will pull the bird's foot. Jiret is a method of hunting for ground layer bird that uses nylon nets then making fences around them using $30 \mathrm{~cm}$ wooden twigs. Usually using cricket/ant as bait. When the ground layer bird eats the bait, the bird's feet will immediately be tied to the nylon rope.
Table 4. Folk classification of birds based on the respondents' knowledge

\begin{tabular}{lcc}
\hline Folk classification & $\begin{array}{c}\text { Species } \\
\text { number }\end{array}$ & $\begin{array}{c}\text { Percentage } \\
\text { of the total }\end{array}$ \\
\hline Distinctive voice/vocalization & 13 & 16.45 \\
Special color & 11 & 13.92 \\
Body size & 6 & 7.59 \\
Special habitat and nest & 7 & 8.86 \\
Distinctive behavior/habit & 4 & 5.06 \\
Morphological characteristics & 4 & 5.06 \\
Feeding guild/role in ecosystem & 2 & 2.53 \\
Others & 32 & 40.50 \\
Total & 79 & 100.00 \\
\hline
\end{tabular}

Table 5. Source of the respondents knowledge on birds

\begin{tabular}{lcc}
\hline Source of knowledge & $\begin{array}{c}\text { Respondent } \\
\text { number }\end{array}$ & $\begin{array}{c}\text { Percentage of the } \\
\text { total }\end{array}$ \\
\hline Parents & 50 & 59.52 \\
Personal experiences & 24 & 28.57 \\
Friends & 9 & 10.71 \\
Total & 84 & 100.00 \\
\hline
\end{tabular}

Jaring or nets are used to catch birds alive, usually used for hunting groups of birds. The bird hunter usually uses this method to hunt Pigeon birds, water birds, or small birds. This hunting method uses nylon nets stretched 100$120 \mathrm{~m}$ in the fields, forest areas, and river edge. Nets are set using $4 \mathrm{~m}$ high bamboo. Usually, the nets are stretched around the river and can be obtained 5-10 birds in one day. This method is carried out in the morning (06.00 a.m.) by deploying nets in the forest/fields, then left in the evening (06.00 p.m.). The hunter returns to take the birds that have been trapped in the nets. The hunting result with this method is estimated at 5 - 60 individuals of bird.

Pitangkeb is carried out using cage made from rectangular woven bamboo pieces. Usually, it uses bait (e.g., crickets/insects) to attract birds. Pitangkeb is used on the ground surface that has previously been made. Bamboo woven is tilted and held with a piece of wood that has been filled with bait. When a bird enters a bait that has been bound to wood, the wood that supports the webbing will automatically close and lock the bird in the ground. Usually, birds hunted with pitangkeb are ground-layer birds, including Orange-headed Thrush (Geokihlia citrina), Chestnut-capped Thrush (Geeokihlia interpres), and Sunda Thrush (Zoothera anthomedae).

Hunters will usually look for sap or leugeut from the tree and put it in a container. The sap of some plant species, including Hevea braziliensis, Artocarpus heterophyllus, or Artocarpus elasticus are commonly used for making leugeut (Iskandar et al. 2016). Then, hunters look for fruit trees in the forest area, usually Ficus trees. According to Iskandar (2017), the tree branches or bamboo stakes that have been smeared are put in various fruit trees, especially in the fruit season, are often visited by fruit-eating birds or insect-eating birds. Besides, hunters use bait to lure birds to the tree using jontrot (birds that have been tamed to attract other birds from nature to approach them). The jontrot 
birds commonly used are chirping birds, e.g. Geopelia striata, Zosterops palpebrosus, and Aegithina tiphia. Cages containing jontrot birds are hung near tree branches that have been smeared with sap. According to Iskandar (2017), birds chirping in a cage can invite birds to come and perch on the branches which have been smeared by sap. Birds trapped in sap are released using oil and stored in cages for trading. The hunted birds are collected and put in the cage. In the past, it was used for bird keeping in the household. Today, however, trapped birds are commonly traded to the urban bird market (Iskandar et al. 2016).

Bedil is a modern tool for hunting because it uses air rifles to catch birds. Usually, this tool is widely used by people outside the village of Girimukti. Shooting birds is a hobby and fun for them. In addition, their hunting results of birds are also usually consumed.

In conclusion, Ciemas Subdistrict area in CiletuhPalabuhanratu Geopark has a high diversity of birds. There were 121 bird species of 47 families in which 88 species were recorded during the field observation and 79 species were documented through the interviews with respondents. Several species with high conservation status, such as Nisaetus bartelsi and Padda oryzivora, were also found in this area. Village people who reside close to the forest have extensive local knowledge of birds. Based on the village people, birds can be classified based on distinctive voice, color, body size, habitat and nest, habit, morphology, and feeding guild. Based on their knowledge of bird functions and bird utilizations, birds have been perceived by village people have an important role in ecological, socio-culture and socio-economic functions. In the past, the village people had traditional beliefs and myths about birds. As a result, some birds that are considered sacred are traditionally not allowed to hunt, keep, and trade, and those birds are traditionally conserved in the village. Nowadays, however, generally many village people do not believe anymore in myths. Consequently, various birds are extensively hunted and used for meat to be consumed, to keep as life birds in the cages as hobby, and to trade for obtaining cash income of the household. The high diversity of birds is considered as an important aspect in the development of the Ciletuh-Palabuhanratu Geopark, Sukabumi, West Java, including for ecotourism development programs. Therefore, to ensure the success of bird conservation in the village, community involvement is really needed, and local knowledge on birds or ethnoornithology studies that aim to understand people's perceptions about birds and the use of birds among them can help in bird conservation efforts.

\section{ACKNOWLEDGEMENTS}

We thank the Centre Environment Sustainability Science and Rector of Padjadjaran University, Indonesia Padjadjaran through the Academic Leadership Grant (Prof. Erri Noviar Megantara), who have supported this research, expertise, and surveyor team. We would also like to thank PAPSI staff (Paguyuban Alam Pakidulan Sukabumi), informants, and respondents of Ciemas Subdistrict, Sukabumi, West Java for providing valuable information.

\section{REFERENCES}

Albuquerque UP, de Lucena RFP, Neto EMFL. 2014. Selection of Research Participants. In: Albuquerque UP, Cruz da Cunha LVF, de Lucena RFP, Alves RRN (eds). Methods and Techniques in Ethnobiology and Ethnoecology. Humana Press, New York.

Alves RRN, Leite RCL, Souto WMS, Bezerra MMD, Loures-Riberio A. 2013. Ethno-ornithology and Conservation of wild birds in Semi-Arid Caatinga of Northeastern Brazil. Ethnobiol Med 9 (14): 1-12. DOI: 10.1186/1746-4269-9-14.

Alves RRN, Souto WMS. 2015. Ethnozoology: A brief introduction. Ethnobiol Conserv 4 (1): 1-13. DOI: $10.15451 /$ ec2015-1-4.1-1-13.

Alves RRN. 2012. Relationships between fauna and people and the role of ethnozoology in animal conservation. Ethnobiol Conserv 1 (2): 1-57.

Andriany SS, Rosana MF, Hardiyono A. 2016. Ciletuh Geopark Geotourism: Geotrack surrounds the beauty of the Ciletuh Mega Amphitheater (The Magical of Ciletuh Amphitheater). Bull Sci Contrib Geol 14 (1): 75-88. [Indonesian]

Berkes F. 2012. Sacred Ecology. 3rd ed. Routledge, New York.

Bibby CJ, Burgess ND, Hill DA. 1992. Bird Census Techniques. Academic Press Limited, London.

Creswell JW. 2009. Research design: qualitative, quantitative, and mixed methods approach. SAGE Publication, Inc. California.

Dinanti RV, Winarni NL, Supriatna J. 2018. Vertical stratification of bird community in Cikepuh Wildlife Reserve, West Java, Indonesia. Biodiversitas. 19 (1): 134-139. DOI: 10.13057/biodiv/d190120.

Hakim L, Andoellah OS, Parikesit, Withaningsih S. 2020. Impact of Agricultural Crop type and hunting on bird communities of two villages in Bandung, West Java, Indonesia. Biodiversitas 21 (1): 5766. DOI: $10.13057 /$ biodiv/d210109.

Haryanto I, Sudjradjat A. 2018. On the Geomorphology and Tectonic Position of Ciletuh-Jampang Area, West Java, Indonesia. Universal J Geosci 6 (2): 47-54. DOI: 10.13189/ujg.2018.060203.

Husodo T, Mochtan KP, Shanida SS, Aminudin SF, Wulandari I, Putra IS, Megantara EN. 2020. Avian diversity in geothermal power plant areas: Case studies in Kamojang, Darajat, and Gunung Salak, West Java, Indonesia. Biodiversitas 21 (3): 1049-1059. DOI: $10.13057 /$ biodiv/d210327.

Husodo T, Shanida SS, Febrianto P, Pujianto MP, Megantara EN. 2019. Mammalian diversity in West Java, Indonesia. Biodiversitas 20 (7): 1846-1858. DOI: 10.13057/biodiv/d200709.

Iskandar J. 1980. Study on Bird Ecology in several villages of Citarum Watershed. Department of Biology, FMIPA, Padjajaran University, Sumedang. [Indonesian]

Iskandar J, Iskandar BS, Partasasmita R. 2016. The local knowledge of the rural people on species, role and hunting of birds: Case study in Karangwangi Village, West Java, Indonesia. Biodiversitas 17 (2): $435-446$.

Iskandar J. 2012. Ethnobiological and Sustainable Development. Research Center for Public Policy and Territorial, University of Padjadjaran, Sumedang. [Indonesian]

Iskandar J. 2016. Ecology of birds in the countryside: A case study in the Citarum River Basin in West Java. Proceeding National Conference and Observer of Birds Indonesia. Atma Jaya University, Yogyakarta, 4-7 Februari 2016. [Indonesia]

Iskandar J. 2017. Ornitologi dan Etnoonitologi. Plantaxia, Yogyakarta. [Indonesian]

Iskandar J, Iskandar BS. 2021. Ethnoornithology: Bird and People. ITB Press, Bandung [Indonesian].

IUCN [International Union for Conservation of Nature]. 2021. The IUCN Red List of Threatened Species Version 2021-1. https://www.iucnredlist.org/. [July 2, 2021]

Krisanti AA, Choirunnafi A, Septiani NO, Pratama FW, Amelia F, Manjaswari A, Septiningtyas PA, Wati AS, Satria JY, Ani IL, Wibowo T, Sugiyarto S. 2017. The diversity of diurnal bird species on western slope of Mount Lawu, Java, Indonesia. Biodiversitas 18 (3): 1077-1083. DOI: 10.13057/biodiv/d180327.

Lynch SJF, Hoelnsteiner RM, Cover CL. 1974. Data Gathering by Social Survey. Philippine Social Science Council, Quezon City. 
Madani A. 2020. Local Knowledge on Vernacular Names, Classification, and Utilization of Birds. Department of Anthropology, University of Padjadjaran, Sumedang. [Indonesian]

Maas B, Tscharntke T, Saleh S, Putra DD, Clough Y. 2015. Avian species identity drives predation success in tropical cacao agroforestry. Appl Ecol 52: 735-743. DOI: 10.1111/1365-2664.12409.

Mackinnon J, Phillipps K, van Balen B. 2010. The birds in Sumatra, Java Bali and Borneo. Center for Biology, LIPI, Bogor. [Indonesian]

Newing H, Eagle CM, Puri RK, Watson CW. 2011. Conducting Research in Conservation: A Social Science Perspective. Routledge. London.

Pam GA. 2017. Ethnoornithology and Conservation: Traditional Ecological Knowledge (TEK) of Birds Among the Conservation of Dilu Forest in Mughere, Palteau State Nigeria. [Thesis]. University of Oxford, UK

Partasasmita R, BS Iskandar, S Nuraeni, J Iskandar. 2019. Impact of the green revolution on the gender's role in wet rice farming: A case study in Karangwangi Village, Cianjur District, West Java, Indonesia. Biodiversitas 20 (1): 23-36. DOI: 10.13057/biodiv/d200104.

Partasasmita R, Shanida SS, Iskandar J, Megantara EN, Husodo T, Parikesit, Malone N. 2016. Human-leopard conflict in Girimukti
Village, Sukabumi, Indonesia. Biodiversitas 17 (2): 783-790. DOI: 10.13057/biodiv/d170255.

Philpott SM, Soong O, Lowenstein JH, Pulido AL, Lopez DT, Flynn DF, DeClerck F. 2009. Functional richness and ecosystem services: bird predation on anthropods in tropical agroecosystems. Ecol Appl 19 (7): 1858-1867. DOI: 10.1890/08-1928.1.

Puri RK. 1997. Hunting Knowledge of Penan Benalui of East Kalimantan Indonesia. [Dissertation]. Department of Anthropology, University of Hawai'i, Hawai'i.

Rheindt FE, Eaton JA. 2010. Biological species limits in the Banded Pitta, Pitta guajana. Forktail 26: 86-91.

Rimanang A. 2016. Pranata Mangsa Ancient Javanese Astrology. Kepel Press, Yogyakarta. [Indonesian]

UNEP-WCMC (Comps.) 2021. Checklist of CITES species. CITES Secretariat, Geneva, Switzerland and UNEP-WCMC, Cambridge. http://checklist.cites.org/

Sekercioglu CH. 2012. Bird functional diversity and ecosystem services in tropical forests, agroforests and agricultural areas. J Ornithol 153 (1): 153-161. DOI: 10.1007/s10336-012-0869-4. 